\title{
X-Ray Crystal Analysis
}

\author{
By Sir William BragG, D.M., F.R.S., Fullerian Professor of Chemistry, Royal Institution, \\ and Director of The Davy-Faraday Research Laboratory
}

$\mathrm{T}$ $\mathrm{HE}$ development of the analysis of crystal structures by means of X-rays has fallen entirely within the period which the citizens of the British Commonwealth are now reviewing. Soon after the beginning of the present reign, a group of German seientific workers made the primary and fundamental discovery. But although the first step was taken abroad, the immediate and consequent advances were made in England; and throughout the whole twenty-five years British workers have taken a full share in the development of a subject which has now grown to extraordinary magnitude. The story of it falls happily, therefore, on the ears of those who now celebrate the King's Jubilee and consider the advances in knowledge which have been made during his reign.

The first experiments were made in Munich in 1912 and have been described in a fascinating manner by Friedrich, who helped to conduct them ; his account is to be found in Die Naturwissenschaft in for April 22, 1922. As has been pointed out by W. L. Bragg ("The Crystalline State", p. 271) the conditions in Munich at that time were peculiarly favourable to the event which gave rise to a new branch of science. Laue, a great mathematician, was profoundly interested in interference phenomena, and Sommerfeld, a great mathematical physicist, in the nature of X-rays and their excitation by the stopping of cathode rays, while Groth was the world's most famous authority on crystallography. The actual incident which precipitated the discovery was a doctor's dissertation by Fwald, who at that time had undertaken, under Sommerfeld's guidance, the study of the passage of light waves through a crystal, considered as a regular arrangement of scattering atoms. When discussing Ewald's problem, Laue was led to ask what would happen if the waves were so short that the wave-length was less than the interatomic distances in the crystal. He realised that spectra should be formed and that waves so short as to be diffracted in this way would be X-rays. An informal discussion on the possibility of observing this phenomenon took place after one of the colloquium meetings and Friedrich, who was then Sommerfeld's assistant, volunteered to carry out some experiments. Success came after several failures. Friedrich gives a vivid picture of his feelings when he saw the diffraction picture in the developing dish.
At that time the writer of this review was contending strongly that the $\mathrm{X}$-rays must be regarded as corpuscular, if their general behaviour, and in particular their relations to cathode rays, were to be explained systematically. It did not occur to him at that time that undulatory and corpuscular theories could be held and worked simultaneously. Consequently, the new experiment seemed to be fatal to his views. It is now understood, of course, that the two theories must go in double harness, even if they do not always seem to pull together.

Naturally perturbed at the apparent contradiction, the writer suggested to his son, who had just completed his course at the University of Cambridge and was looking for a problem to attack, that he should study the new experiment and see if any reconciliation could be found. The conclusion that Laue was essentially correct was soon found to be inevitable. At the same time, it appeared to the young worker that Laue's explanation of the details of his zinc blende photographs was unnecessarily complicated. He found it simpler to approach the problem with the idea that electromagnetic pulses, equivalent to white light' in the X-ray region, were reflected from the crystal planes. This was suggested by the behaviour of the spots in the photograph when the crystal was tilted and by their shape. He was then able to draw certain conclusions as to the structure of zinc blende. This result was published in November 1912. The Laue photographs of sodium chloride and potassium chloride were found to be even simpler than that of zinc blende, so that ail the details of their structures could be found. These two were the first crystals to be analysed.

Meanwhile, the writer at Leeds, and Moseley and Darwin at Manchester, examined the reflected beams of X-rays, using ionisation chamber and absorbing screens, and found that they had all the characteristic properties of the incident beam. From these researches came the $\mathrm{X}$-ray ionisation spectrometer, which showed not only the 'white' radiation reflected over a wide range of angle but also the peaks of the characteristic line spectrum of the material of the target in the $\mathrm{X}$-ray tube.

The discovery of the line spectrum had two results. In the first place, it made crystal analysis enormously more powerful. Several fairly simple structures were quickly solved by its means, such 
as zinc sulphide, iron pyrites, fluorspar and calcite. In the second place, the high-frequency spectra of the elements could be measured accurately. This led to the brilliant and well-known generalisation of Moseley who, by using an extended range of anticathodes, was able to formulate the laws relating frequency to atomic number. Moseley's death at Gallipoli in 1915 was a sad blow to the development of a new science.

The outbreak of War in 1914 put an end to most of the researches in crystal analysis then proceeding. In the two short years great progress had, however, been made. The determination of crystal structure had begun. The ionic, or heteropolar, character of many crystals had been discovered. It is to be observed that in some of these early determinations the $\mathrm{X}$-rays were called on for nothing more than a simple and ready decision between structures already suggested by other considerations. But the decision was final and very helpful. The measurements of the intensities of the rays reflected from the various crystal planes had not yet come to play the important part that it does to-day. The measurements were used to determine the geometrical quantities of the crystal, the size of the unit cell and a few important data as to the relative positions of the elements in it. Intensities were measured in certain cases, but no great accuracy was required. Indeed, the technique was barely able to supply it. Moreover, there was the difficult question of the interpretation of the meaning of intensity measurements. Darwin had, indeed, covered almost the whole ground of the theory of X-ray reflection. $\mathrm{He}$ distinguished between the perfect crystal, which is indeed a rarity, and the so-called 'mosaic crystal', which consists of an assemblage of small and more perfect crystallites, mutually aligned with greater or less accuracy. But his results were not put to their full use.

In some of the neutral countries work was carried on, and important results were obtained. In Sweden, Siegbahn developed a fine school devoted to spectrum measurements in which extraordinary accuracy was obtained. In Switzerland, Debye devised the 'powder' method which could be applied to the analysis of materials so finely subdivided that the crystalline character could not be observed even with the aid of the microscope. The crystalline character of colloidal suspensions, for example of gold, was a discovery of great interest. In America, Hull made use, independently, of the same method. The consequence of this advance was the inclusion of a vast number of substances, hitherto not recognised as crystalline, within the range of the X-ray analysis.
As soon as the laboratories could settle down to work again, after the War was over, the new subject was energetically studied all over the world and progress was rapid. It was natural that in the first place efforts should be devoted to the task of shaping a new crystallography. The old had been founded almost entirely upon the external characteristics of crystals; the new gave insight into the body of the crystal and was obviously much more fundamental. The dis. coveries that had already been made gave promise of various useful generalisations, and these called for careful examination. Three main types of structure were to be recognised. They may be illustrated from the work done before the War. There was the heteropolar type represented by rocksalt, in which ions were arranged so that each ion of one sign was surrounded by a certain number of ions of the contrary sign, all equally related to it. There was the homopolar type represented by diamond, in which the atoms were held together by the valency bonds of the chemist, now called co-valent bonds. In the diamond and other structures such bonds linked the crystal together into one whole. We know now that in most organic crystals only the atoms of each molecule are so linked together, the ties between molecule and molecule being the comparatively weak forces of Van der Waals. Lastly, there were the metals such as, for example, aluminium, in which the atoms were held together by what may be termed an electronic cement.

In all these types one common and most im. portant feature at once emerged. The atoms or ions retained their characteristic dimensions in whatever structure they were embodied. The first tables of atomic or ionic radii were drawn up by W. L. Bragg, and in Finland by Wasastjerna. On these considerations Goldschmidt and his assistants reared a 'geochemistry' which showed how largely the various forms of crystal structure were governed by rules of geometry. To Niggli we owe the idea of a systematic deduction of the 'space-group' by means of X-ray data.

The most important constituents of the earth's crust are the silicates. Their immense variety of structure and chemical constitution had long presented an analytical problem which seemed to be insoluble by the older methods. W. L. Bragg and his colleagues at Manchester have been successful in resolving their complications. The oxygen atoms, it seems, play a leading part in the structure. They form the bricks, laid as regularly as possible ; while the other constituent atoms, used somewhat indiscriminately, bind the whole together. In the course of this work it has been necessary to 
measure the intensities of the reflections from the various crystal planes, determining their values relative to the primary rays. The electron distributions within the atoms also required evaluation, for which the theoretical work of Hartree and the experimental work of James and others proved invaluable. Also, at the University of Manchester a study of alloys has been very successful, Bradley's determinations of $\gamma$-brass and $\alpha$-manganese leading the way. At the present moment this branch of the subject is developing rapidly; by its means new insight is being gained into many metallurgical problems. To the study of alloys, Westgren in Sweden contributed much pioneering work. Another outstanding contribution has been Hume-Rothery's theory that the electron-atom ratio is a major factor in determining the type of an alloy structure.

Crystals composed of the complicated molecules of organic substances require special treatment if complete solutions are aimed at. It can be shown that every reflection which a crystal yields implies a harmonic variation of electron density, the magnitude of which corresponds to the intensity of the reflection. By measuring the absolute intensities of one or two hundred reflections, and summing up the implied harmonic variations in the manner of a Fourier series, a picture of the distribution of electron density is obtained. The result is usually displayed in the form of a contour map, showing the densities projected upon some principal plane. The atoms are clearly outlined and their positions can usually be determined with great accuracy. Very interesting determinations have been made in this way by J. M. Robertson, Iball, Miss Knaggs in the Davy-Faraday Laboratory, by Wyckoff in America and others. Here again there emerges a remarkable constancy in the distances between atomic centres : given a pair of atoms and the character of the bond, single, double or treble, the distance that separates them seems to be always the same, in whatever structure they enter. In this case there is an added constancy in the orientations of the bonds in general: though in special circumstances variations may be forced upon them.

One of the most remarkable of the organic substances is the carbon chain which is the essential part of the paraffins, fatty acids, alcohols and the like. Its complete elucidation is due to Müller, Shearer and Piper in Great Britain, to M. de Broglie, Friedel and Trillat in France, and to others. The frequency with which the long chain appears in the construction of Nature is extraordinary and suggestive. It may be all of carbon, as in the cases just quoted: or it may include nitrogen as in the proteins, or as in cellulose it may consist of a series of linked rings each formed of carbon and oxygen.

Some of the most remarkable investigations in organic substances have been made by Bernal, his subject being the vitamins and other biological structures. In this way the $\mathrm{X}$-ray analysis is now contributing to biology.

No one expected, when the new study began, that such a complicated substance as cellulose would have yielded to treatment : but Mark, and Polanyi in Germany, and many others have found it possible to gain much useful information from the X-ray photographs, ill-defined as they are in comparison with those of crystals that can be recognised as such. This work was, of course, inspired by the great importance of cellulose in many industries. Later, Astbury, at Leeds, was able to solve the curious problem of the extensibility of keratin and other proteins. He was able to show, for example, why wool is elastic and silk is not, basing his explanation on the determination of their fundamental structure. Recently he has explained in the same way the extensibility of nerve and musole.

Naturally the determination of crystal structure has given a great opportunity to the mathematical physicist to formulate the laws which govern it, and to trace their actions. The new system of wave-mechanics introduced by de Broglie and Schrödinger has been brought to bear, and much interesting work has already been done. To this Born, Hund, Hückel in Germany, Hartree, Fowler, Lennard-Jones in England, Compton, Pauling and Slater in America, and many others in all parts of the world have made contribution.

Lastly, a word must be said in respect to the technical applications of X-ray analysis. These have been many and varied. A section of the staff of the National Physical Laboratory under Shearer has been engaged in the study of various problems submitted by industry. A vast amount of research has been carried out in various places on the deformation of metal crystals, and the alterations in structure and properties due to coldworking. In this Polanyi has been the pioneer. In England, G. I. Taylor has been a chief contributor, while Gough at the National Physical Laboratory has concerned himself chiefly with the special question of the nature of metal fatigue.

This short sketch does but touch on a few of the principal matters that have formed part of the development of a subject now grown to very large dimensions; and it has only been possible to refer by name to a few of the many who have contributed thereto. 